of adult patients in whom pretreatment CT images and subsequent WHO 2016 diagnoses of infiltrating glioma were available. Presence and pattern of tumor calcification were determined by consensus. RESULTS: A total of 492 patients met inclusion criteria. Median age was 59 years and $61.8 \%$ were male. Tumor types included glioblastoma IDH wild-type (IDHwt) in $272 / 492$ patients $(55 \%)$, glioblastoma IDH mutant (IDHmut) in $18 / 492$ (4\%), WHO grade II/III astrocytoma IDHwt $(71 / 492 ; 14 \%)$, WHO grade II/III astrocytoma IDHmut (56/492; 11\%), and WHO grade I//III oligodendroglioma $(69 / 492 ; 14 \%)$. The remaining six tumors $(1 \%)$ could not be full characterized. Tumor calcification was present in $47 / 492$ patients $(10 \%)$. The majority of patients with calcification $(27 / 47 ; 57 \%)$ were diagnosed with WHO grade II or III oligodendroglioma, followed by glioblastoma IDHwt $(8 / 47,17 \%)$. The presence of calcification was significantly associated with IDH mutation and $1 \mathrm{p} / 19 \mathrm{q}$ codeletion (each $\mathrm{p}<0.001$ ). A pattern of calcification we termed "classic varicoid" was observed in 18 patients (16 oligodendroglioma, one IDHmut GBM, and one IDHmut astrocytoma). Only 14/343 (4\%) of patients with IDHwt WHO grade II/III astrocytoma or IDHwt glioblastoma demonstrated calcification and none showed the "classic varicoid" pattern. DISCUSSION: Tumor calcification at CT strongly predicts oligodendroglial pathology, despite the far greater frequency of astrocytoma/glioblastoma. The "classic varicoid" pattern of calcification is highly specific for IDH mutant gliomas.

NIMG-50. VOLUMETRIC ANALYSIS OF IDH-MUTANT LOW-GRADE GLIOMA: A NATURAL HISTORY STUDY OF TUMOR GROWTH RATES BEFORE AND AFTER TREATMENT

Ray Huang ${ }^{1}$, Robert J. Young ${ }^{2}$, Benjamin Ellingson ${ }^{3}$,

Harini Veeraraghavan ${ }^{4}$, Wei Wang ${ }^{5}$, Tracy Luks ${ }^{6}$, John Kim ${ }^{7}$,

Elizabeth Gerstner ${ }^{8}$, David Schiff ${ }^{9}$, Katherine B. Peters ${ }^{10}$, Ingo

K. Mellinghoff ${ }^{4}$, Susan Chang ${ }^{11}$, Timothy Cloughesy ${ }^{3}$ and Patrick Y. Wen ${ }^{12}$, ${ }^{1}$ Brigham and Women's Hospital, Boston, MA, USA, ${ }^{2}$ Memorial SloanKettering Cancer Center, New York, NY, USA, ${ }^{3}$ University of California at Los Angeles, Los Angeles, CA, USA, ${ }^{4}$ Memorial Sloan Kettering Cancer Center, New York, NY, USA, ${ }^{5}$ Brigham and Women's Center, Boston, MA, USA, ${ }^{6}$ Department of Radiology and Biomedical Imaging, University of California San Francisco, San Francisco, CA, USA, ${ }^{7}$ University of Michigan Health System, Ann Arbor, MI, USA, ${ }^{8}$ Massachusetts General Hospital, Boston, MA, USA, ${ }^{9}$ University of Virginia, Charlottesville, VA, USA, ${ }^{10}$ The Preston Robert Tisch Brain Tumor Center, Duke University Medical Center, Durham, NC, USA, ${ }^{11}$ University of California, San Francisco, San Francisco, CA, USA, ${ }^{12}$ Dana-Farber/Harvard Cancer Center, Boston, MA, USA

INTRODUCTION: Low-grade gliomas, particularly those carrying IDH1/2 mutations, often demonstrate long survival times making evaluation of treatment efficacy difficult. We investigated the volumetric growth rate of IDH mutant gliomas to determine whether a significant change in growth rate before and after treatment can be detected, thereby establishing this imaging approach as a response endpoint in low-grade glioma trials. METHODS: In this multicenter study, 241 adult patients who had progressive nonenhancing gliomas (WHO grade II or III) with IDH1 or IDH2 mutation were retrospectively identified. Subjects were required to have at least 3 MRI scans containing T2/FLAIR imaging spanning a minimum of 6 months prior to treatment. A subset of 100 patients who received chemotherapy, radiation therapy, or chemoradiation therapy and had more than 2 post-treatment imaging time points available were evaluated for change in pre- and post-treatment growth rates. Semi-automatic segmentation was performed to calculate tumor volume at each time point A mixed-effects model was used to study tumor growth prior to treatment. A piecewise mixed model was used to compare tumor growth rates preand post-treatment. RESULTS: Under an exponential tumor growth mode assumption, the pre-treatment volumetric growth rate for the entire cohort $(\mathrm{N}=241)$ was $0.196 \mathrm{~mL} / 180$ days (95\% CI:[0.168-0.224]) when measured by all available MR scans and $0.296 \mathrm{~mL} / 180$ days (95\% CI:[0.252-0.340]) when measured using scans within one year of treatment initiation. Using MRI measurements within one year before and one year after treatment initiation $(\mathrm{N}=100)$, there was a significant difference in volumetric growth rates before $(0.276 \mathrm{~mL} / 180$ days, $95 \% \mathrm{CI}:[0.194-0.358])$ and after treatment $(-0.156 \mathrm{~mL} / 180$ days, $95 \%$ CI: $[-0.241,-0.071])(\mathrm{p}<0.0001)$. CONCLUSION In this study, we evaluated the growth rates of IDH mutant gliomas to establish the natural history of tumor growth behavior and provide preliminary evidence that changes in tumor volume growth rates can be a meaningfu endpoint of treatment response.

NIMG-51. RADIOLOGIC PHENOTYPES ARE TREATMENT SPECIFIC AND ASSOCIATED WITH SURVIVAL - EXPLORATORY ANALYSIS OF EORTC 26101

Martha Nowosielski ${ }^{1,2}$, Thierry Gorlia ${ }^{3}$, Inga Harting ${ }^{4}$,

Alexander Radbruch ${ }^{5}$, Alba Brandes ${ }^{6}$, Martin J.B. Taphoorn ${ }^{7}$, Walter Taal ${ }^{8}$, Julien Domont ${ }^{9}$, Ahmed Idbaih ${ }^{10}$, Mario Campone ${ }^{11}$, Paul M. Clement ${ }^{12}$, Roger Stupp ${ }^{13,14}$, Michel Fabbro ${ }^{15}$, Emilie Le Rhun ${ }^{16}$, Andreas von
Deimling ${ }^{17}$, Michael Platten ${ }^{1}$, Vassilis Golfinopoulos ${ }^{3}$, Martin Bendszus ${ }^{4}$, Martin van den Bent ${ }^{18}$ and Wolfgang Wick ${ }^{1},{ }^{1}$ University Medical Center \& German Cancer Research Center, Heidelberg, Germany, ${ }^{2}$ Department of Neurology, Medical University Innsbruck, Innsbruck, Austria, ${ }^{3}$ European Organization for Research and Treatment of Cancer Headquarters, Brussels, Belgium, ${ }^{4}$ Department of Neuroradiology, University Medical Center \& German Cancer Research Center, Heidelberg, Germany, ${ }_{5}^{5}$ Department of Radiology, German Cancer Research Center (DKFZ), Heidelberg, Germany, ${ }^{6}$ AUSL-Bologna-IRCCS Scienze Neurologiche, Bologna, Italy, ${ }^{7}$ VU University Medical Center, Amsterdam, Netherlands, ${ }^{8}$ The Brain Tumor Center at Erasmus MC - Cancer Institute, Rotterdam, Netherlands, ${ }^{9}$ Institut Gustave Roussy, Villejuif, France, ${ }^{10} \mathrm{AP}-\mathrm{HP}$, Hôpitaux Universitaires La Pitié Salpêrière - Charles Foix, Service de Neurologie 2 Mazarin, Inserm U 1127, CNRS UMR 7225, Sorbonne Universités, UPMC Univ Paris 06 UMR S1127, Institut de Cerveau et de la Moelle épiniére, ICM, Paris, France, Paris, France, ${ }^{11}$ Institut de Cancerologie de l'Ouest (ICO) - Centre Rene Gauducheau, Saint-Herblain, France, ${ }^{12}$ Universitair Ziekenhuis Leuven, Leuven, Belgium, ${ }^{13}$ Northwestern University Feinberg School of Medicine, Chicago, IL, USA, ${ }^{14}$ Northwestern University, Feinberg School of Medicine, Chicago, IL, USA, ${ }^{15}$ Institut régional du Cancer Montpellier, Montpellier, France, ${ }^{16} \mathrm{CHU}$ Lille, General and Stereotaxic Neurosurgery service; Oscar Lambret Center, Medical Oncology Department, Lille, France, ${ }^{17}$ Department of Neuropathology, Institute of Pathology, German Cancer Research Center, University Heidelberg, Heidelberg, Germany, ${ }^{18}$ Neuro-Oncology Unit, Erasmus MC Cancer Center, Rotterdam, Netherlands

BACKGROUND: EORTC 26101, a randomized phase III trial in recurrent glioblastoma compared lomustine plus bevacizumab to single agent lomustine. In this exploratory analysis the tumors were radiologically followed and characterized with the objective to investigate whether type of radiologic progression differs between the treatment groups and is related to outcome. METHODS: Five progression types (PTs) were categorized using an adapted algorithm according to MRI contrast enhancement behavior in T1 and T2-weighted images in 373 patients (lomustine, $n=129$; combination, $\mathrm{n}=244$ ). Frequencies of PTs (designated as cT1 relapse, classic T1, T2 diffuse, $\mathrm{T} 2$ circumscribed and primary non-responder), time to progression (PFS) and overall survival (OS) were assessed. Frequencies were compared by Wilcoxon rank test, univariate associations of PTs with survival were tested by log-rank test, hazard ratios (HR) with $95 \%$ Confidence interval were calculated by Cox model. RESULTS: Except for "classic T1", frequencies of PTs differed between the treatment arms, with non-enhancing tumor progression (T2 diffuse and T2 circumscribed) being more commonly detected in the combination group. Complete resolution of contrast enhancement (cT1 relapse) during treatment was associated with better survival than mere decrease (classic T1) of contrast enhancement (PFS; HR=0.57 [0.29.;1.11]) and OS; HR $=0.42[0.15 ; 1.18]$ for lomustine, PFS; HR $=0.33[0.23 ; 0.45]$ and OS; HR $=0.37[0.26 ; 0.52]$ for combination group. CONCLUSIONS: Progression of glioblastoma can be characterized radiologically. These radiologic phenotypes are influenced by treatment and are associated with differential outcomes. Complete decrease of contrast enhancement during treatment is associated with longer PFS and OS.

\section{NIMG-52. RADIATION-INDUCED VASCULAR MALFORMATIONS MIMICKING TUMOR IN MRI-BASED TREATMENT RESPONSE ASSESSMENT MAPS (TRAMs)}

Leor Zach ${ }^{1,2}$, David Guez ${ }^{2}$, David Last ${ }^{2}$, Dianne Daniels ${ }^{2}$, Shirley Sharabi ${ }^{2}$, Dvora Nass ${ }^{1,2}$, Ouzi Nissim ${ }^{2}$, Roberto Spiegelmann ${ }^{1,2}$, Galia Tsarfaty ${ }^{1,2}$, Chen Hoffmann 1,2 , Alisa Talianski ${ }^{2}$, Yigal Shoshan ${ }^{3}$, Yakov Fellig', Sagi Harnof ${ }^{1,5}$, Zvi Cohen ${ }^{1,6}$ and Yael Mardor ${ }^{1,2} ;{ }^{1}$ Tel-Aviv University, TelAviv, Israel, ${ }^{2}$ Sheba Medical Center, Ramat-Gan, Israel, ${ }^{3}$ Hadassah Medical Center, Jerusalem, Israel, ${ }^{4}$ Pathology Department, Hadassah Hebrew University Medical Center, Jerusalem, Israel, ${ }^{5}$ Rabin Medical Center, Petach Tikva, Israel, ${ }^{6}$ Department of Neurosurgery, Sheba Medical Center, RamatGan, Israel

BACKGROUND: We have recently presented MRI-based TRAMs enabling efficient separation between tumor (contrast clearance $>1$ hour post injection, blue) and treatment-effects (TEs, contrast accumulation, red), validated histologically in 51 resected patients reaching $92 \% / 100 \%$ $\mathrm{PPV} /$ sensitivity to morphologically active tumor. This high selectivity stems from the ability of viable vessels in blue/tumor regions to efficiently clear contrast by 1 hour, while damaged lumens in red/TEs regions are unable to, resulting in contrast accumulation. $~ 400$ adult patients have been recruited to our studies in Israel since 2010. As the TRAMs cannot differentiate blood vessels from active tumor (both appearing blue), we studied whether radiation-induced cavernoma-like vascular malformations (RICMs) may mimic tumors in the TRAMs. METHODS: Cases in which the pre-surgical TRAMs were found false-positive for tumor were re-reviewed. For comparison, 2 patients with newly diagnosed cavernomas and 4 with treated AVMs were scanned by the TRAMs. One AVM underwent TRAMs-guided biopsies. RESULTS: Four cases of 